\title{
FROM ACTIVE TO PASSIVE: PROGRESS IN TESTING OF INTERNET ROUTING PROTOCOLS
}

\author{
Jianping Wu, Yixin Zhao and Xia Yin \\ Department of Computer Science, Tsinghua University, Beijing 100084 \\ jianping@cernet.edu.cn, \{zhaoyx,yxia\}@csnet1.cs.tsinghua.edu.cn
}

\begin{abstract}
Routing protocols play an important role in the Internet and the test requirements are mounting. To test routing protocols more efficiently, several enhanced techniques are applied in our Protocol Integrated Test System. The Implementation Under Test is modeled as a black box with windows. The test system is endowed with multiple channels and multiple ports to test distributed protocols. The test suite and other related aspects are also extended. Meanwhile, a passive testing is introduced to test, analyze and manage routing protocols in production field, capable of performing the conformance test, the interoperability test and the performance test. Necessary algorithms and techniques were worked out for the passive testing to cover the scope from the protocol state machine to the routing information manipulation. This paper presents our efforts to test Internet routing protocols with both the active testing and the passive testing.
\end{abstract}

Keywords: routing protocol, test structure, passive testing

\section{INTRODUCTION}

High performance and stable routing protocols dominate the performance of the Internet. Currently the most commonly used routing protocols are RIP [1], OSPF [2] and BGP [3]. There are other routing protocols like [4-6], but despite their importance, the test activity is still rather limited due to the fact that the original test theories, structures and systems were aimed at communication protocols. While routing protocols possess different features. 
Several deficiencies were encountered when we started our work on routing protocol test with the Protocol Integrated Test System - PITS [7].

Then the Implementation Under Test (IUT or module under test MUT) is modeled as a windowed black box and tested with a multiple channel test method. The test suite has evolved into a heterogeneous one, while the test data of different types can be described in their appropriate formats. Enhanced techniques mainly focus on the Reference Implementation (RI). In cooperation with the Enhanced RI (ERI), the packet extension technique helps to describe the whole test activity integrally and uniformly.

Some abnormalities will only appear in practice and/or over a long time, such as route oscillations [8], useless route advertisements [9] or even exhausted routers. Although the active testing helps a lot to uncover the deficiencies of the implementations of routing protocols [10] [11], it can hardly deal with these problems. The passive testing [12] can be performed in production field over a long period of time without interference on the network. It is able to perform the conformance, interoperability and performance tests. Therefore, the passive testing has also been adopted in our work on routing protocol test. Corresponding techniques have also been worked out [13][16].

All the following methods are applied in the test activity. We have built an Enhanced Reference Implementation [19] for PITS An intelligent online BGP-4 analyzer[20] has been implemented and then a prototype of a more general online test system [21]. Another important advance is that the active testing and the passive testing are combined, which helps us in the effort to cover all features of routing protocols in our test. These techniques are applicable for RIP, OSPF and BGP. In this paper, we will mainly focus on BGP-4 as the example.

\section{FEATURES AND TEST REQUIREMENTS OF ROUTING PROTOCOLS}

Compared with communication protocols such as TCP/IP, routing protocols are quite different. First, the purpose of the routing protocol is to collect the information needed to do routing [1], which is a basic summary of routing protocol functions. Another extraordinary character is the nature of distributed systems, which can be found from both the interaction between different routers and the communication between the interfaces of one single router. No matter the designation or the implementation, there are several important databases to store the routing information. The information flow among these databases is connected by the internal processes which dominate the correctness and efficiency of routing protocol implementations. 
For a routing protocol, there is no "upper level protocol" at all. However the interaction of routing protocols is much more complex. For example, BGP-4 will talk with neighbor peers while exchanging information with other routing protocols such as OSPF on the same router. A more detailed discussion can be found in [14]. For the testing of communication protocols, the protocol state machine is heavily relied upon. The state machines only describe a small part of the behavior of routing protocols. The majority of a routing protocol activities begins after it enters a "stable" state (e.g. established state for BGP-4).

Therefore, the testing of routing protocol should cover (1) the packet structure, (2) the protocol state machine, (3) the interoperability, (4) exchange and manipulation of routing information, (5) performance evaluation and (6) other features such as the policy-based routing and the route flap damping.

\section{CURRENT TEST SYSTEM AND DEFICIENCIES}

\subsection{Structure of PITS}

PITS is the second generation of the test system we developed. Figure 1 shows the structure of PITS. PITS exploits test suites in TTCN[15] format and adopts a concurrent interpretive test execution method for high efficiency. The test suite generation tool TUGEN generates a TTCNformatted test suite from the formal description of the protocol. Test Execution (TE) is the key engine in the test process, responsible for test process control and the generation of all the information needed in the test report. PITS is a general tester, that is, TE works independently of any particular protocol. All the protocol-related information is reflected in the test suite and the corresponding RI.

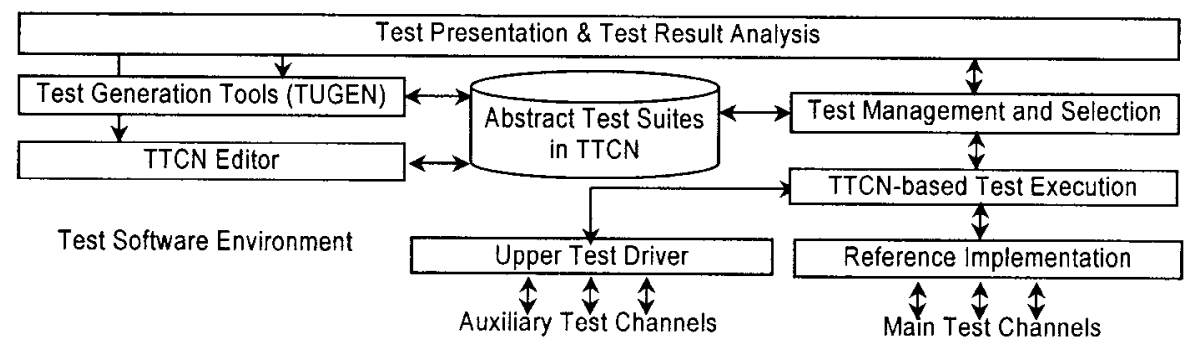

Figure 1 Protocol Integrated Test System 


\subsection{Deficiency and limitation of current test system}

Since PITS was mainly aimed at the testing of communication protocols, some deficiencies were uncovered when testing routing protocols.

Originally, RI works in a very simple way, communicating with TE to get commands and data and sending/receiving data packets through the service provider. Test data and correct results are written in the test suite in advance and it is up to TE to accomplish the final comparison and judgement. Since there are large amounts of data transmission and routing information processing and TE depends on interpretive means to execute, TE can hardly accomplish the numerical calculation.

In traditional work, the test system only sets up a communication channel with the MUT and always exploits only one port. With such a test structure, only the packet structure and the protocol state machine can be tested. Since the routing protocol possesses features of distributed systems, the test system must be able to provide multiple ports. Only then can routing information exchange and processes be tested. Even if multiple ports are available, the entire scope of the testability is restricted to the data that the routing protocol module sends out. In addition, testing the correctness of the routing protocol under different conditions is also very important, especially for policy-based routing. Manual configurations are not only timeconsuming but also error prone. On the other hand, the routing protocol is always implemented in the router while interacting with other modules. These modules provide the information and the access to the routing protocol module and the whole router through standard interfaces. In the original test structure, since the test system only connects with the MUT, this information and access can't be exploited at all.

There are further limitations regarding TTCN. It is difficult to describe certain cases with TTCN and it is almost impossible for TE to execute it. We believe that the latest TTCN-3 has greater ability and flexibility. However, the necessary editor, the executing environment, is under development.

The router always has a powerful processing ability while the speed of the test system is relatively much slower. When the test case shown in figure 2 is executed, there may be possible packet loss.

\begin{tabular}{|c|c|c|c|c|}
\hline No & Behavior Description & Label & Constraints Ref & Verdict \\
\hline & $\ldots$ & & & \\
\hline 1 & T! OPEN_PACKET & & C1_OPEN & \\
\hline 2 & START T_HOLD & & & \\
\hline 3 & T? KEEPALIVE_PACKET & & C1_KEEP & \\
\hline 4 & $\ldots$ & & & \\
\hline 5 & $?$ TIMEOUT & & & FAlL \\
\hline 6 & T? OTHERWISE & & & FAIL \\
\hline & $\ldots$ & & \\
\hline
\end{tabular}

Figure 2 The test case with the possibility of packet loss 
When step 1 is interpreted, the data and the command are sent to RI. RI then sends out the packet OPEN_PACKET. MUT will respond with a KEEPALIVE_PACKET. Since the speed of the router is rather fast, this packet is sent back immediately. At that time, if step 3 hasn't finished its interpretation RI will not accept the response packet. Further, when step 3 is done and RI is informed to receive some packet, what RI actually receives may be some other packet and step 6 will then be entered. The other scenario is that RI receives nothing at all. When the timer T_HOLD expires, step 5 will be entered.

\section{ENHANCING TECHNIQUES}

In order to solve these problems discussed above, the test system needs to be enhanced accordingly. After some analysis, almost all the enhancements are related to RI.

\subsection{Functional sub-modules introduced into RI}

In order to release TE from the heavy burden of the calculation, the dynamic calculation and the data generation sub-modules are introduced (shown in figure 3).
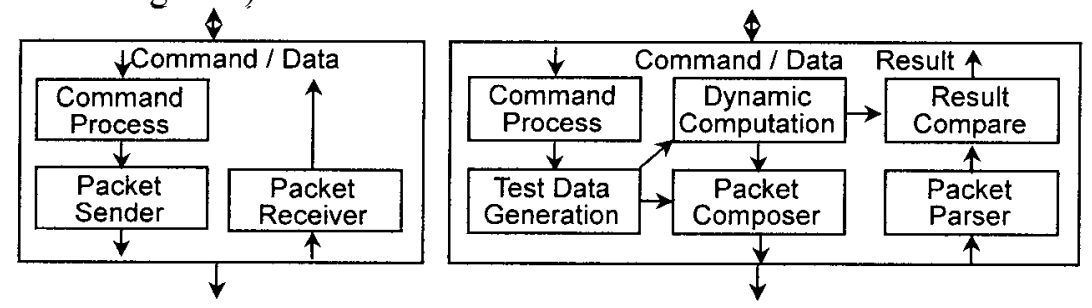

Figure 3 RI and enhanced RI with functional sub-modules

The test data generation sub-module in the ERI selects the test data or generates new test data with some algorithm according to the demand of TE. The packet composer sub-module forms the data packet and sends them out while the dynamic computation sub-module calculates the expected results. Response from the IUT (or MUT) is first processed by the packet parser submodule, after which the results are compared with what are expected and finally presented to TE. Through these means, ERI accomplishes the calculation originally taken by TE, which improves the efficiency of the test execution. 


\subsection{Windowed black box model and multiple channels test method}

In order to take full advantage of the information and access provided by the system (for example, a router) where the MUT resides, the windowed black box model and the multiple channel test method are adopted. The windowed black box takes into consideration not only the MUT but also other modules interacting with it. These modules offer standard interfaces and provide the tester with several windows to enhance the observation and the control of the MUT in the routing protocol test. Accordingly, the multiple channel test method is exploited. In traditional work, the test system only sets up a communication channel with the MUT, we call this channel a "basic channel", as in [10]. The principal difference of the multiple channel test method is that not only does the test system set up a communication channel with the MUT, it also sets up channels with other modules interacting with it (shown in figure 4).

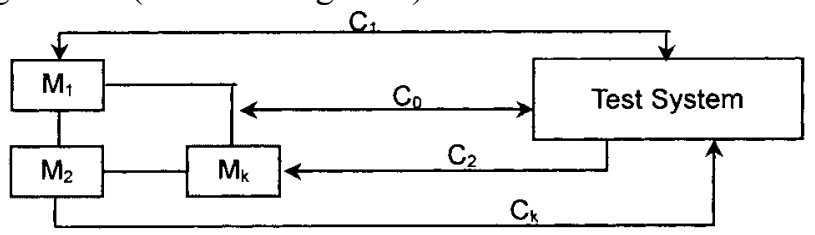

Figure 4. Multiple channels test method and test structure

The $\mathbf{C}_{0}$ is the basic channel between the test system and the MUT. $\mathbf{M}_{1}, \mathbf{M}_{2}, \ldots, \mathbf{M}_{\mathbf{k}}$ are the $\mathrm{k}$ modules (will have been tested in advance) interacting with the MUT. The test system sets up channels $\mathbf{C}_{\mathbf{1}}, \mathbf{C}_{\mathbf{2}}, \ldots, \mathbf{C}_{\mathbf{k}}$ with these modules. The main idea of the multiple channel test method is to take full advantage of the information available to enhance the observability and the controllability, thus promoting the test efficiency and the coverage. The multiple ports needed to test distributed protocols can be easily implemented by expanding the channels.

\subsection{Partition of acceptance sub layer and submission sub layer}

In order to avoid possible packet loss in high-speed networks, RI is partitioned into two sub layers: the acceptance sub layer and the submission sub layer. The acceptance sub layer takes charge of receiving packets from the MUT, stores them in a buffer and maintains them. The submission sub layer fetches the packets and provides them to TE according to its demand. The acceptance sub layer makes RI independent of the speed of the lower 
network and the packet loss can be avoided by managing a proper interval between test case execution.

\subsection{Relation of enhanced techniques}

The structure of the ERI is shown in figure 5, which also explains the relation of these enhancing techniques. The acceptance and the submission sub layers reside at the bottom of the ERI. All the sub layers above can take advantage of them to make up for the speed gap. In the middle of the ERI structure, are basic and extended channels. Each channel can provide multiple ports in order to test distributed routing protocols. Meanwhile, each channel possesses functional sub-modules such as dynamic calculation and data generation (details can be referred to in figure 3) which can provide more processing ability and flexibility. The topside of the ERI contains the command/data dispensation and collection sub-module, communicating with $\mathrm{TE}$, getting data/command, dispensing it to corresponding channels and returning the result to TE for the final verdict.

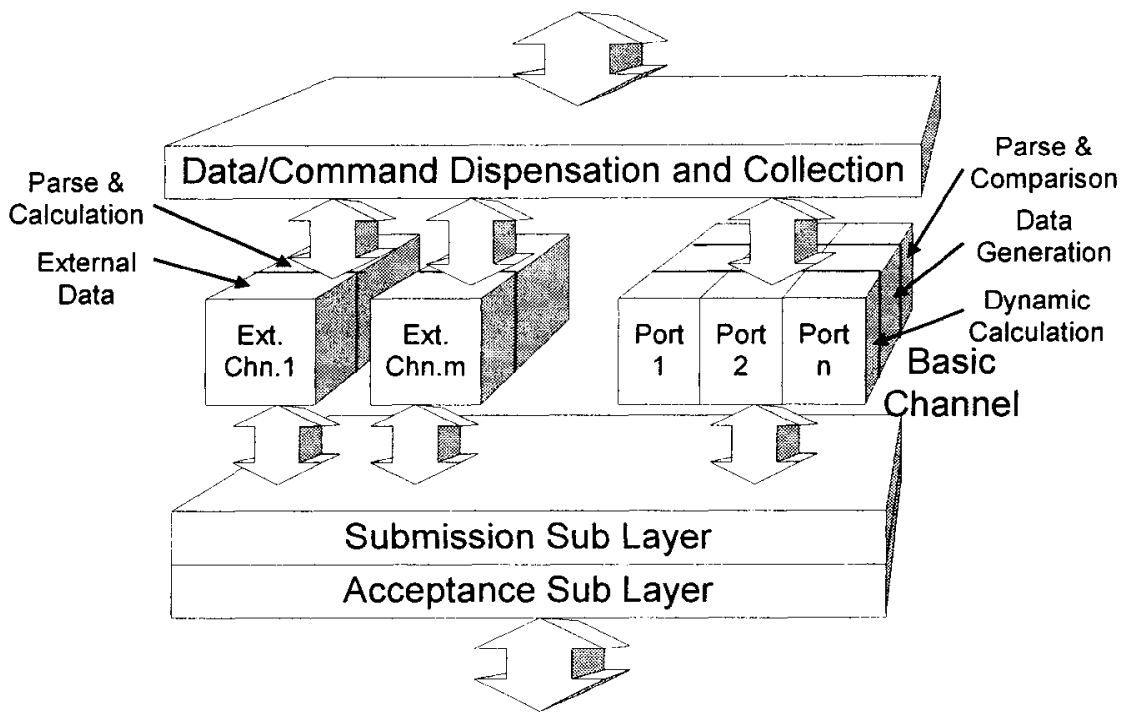

Figure 5 Structure of the Enhanced Reference Implementation

It can be seen that the ERI overcomes the problems discussed above and possesses these features: (1) functionally compatible with the former RI; (2) no effect on the syntax and semantic integrity of TTCN; (3) provides multiple channels to increase the observability and the controllability of the MUT; (4) multiple ports to test distributed routing protocols; (5) reduces the burden of TE and improves the flexibility of PITS. (6) enhanced functionality suitable for the routing protocol test. 


\subsection{Describing the test activity in the test suite integrally and uniformly}

The multiple channel test method also brings new problems. One is how to describe the test activity uniformly and integrally. Therefore, the packet expansion technique is raised. For example, four types of BGP-4 packets are defined in [3] and we defined type 128-143 for the data sent through the configuration channel and 144-161 for the auxiliary information channel. This definition does not contradict current usage of packet types and is unlikely to cause future conflicts. However, there is no actual data in the date field of the packet with special type values. Instead, there is only an index. Corresponding channels will look up the data in their mapping tables. This form of representation is a natural extension of the PIXIT (Protocol Implementation eXtra Information) which can act as an external part of the test suite.

\section{APPLIED PASSIVE TESTING IN ROUTING PROTOCOL TEST}

\subsection{Brief of passive testing}

Some abnormalities will only appear in practice and/or over a long time, such as route oscillations [8], useless route advertisements [9] and sometimes even exhausted routers. Although active testing can do a lot to promote the quality of the network equipment, it can hardly deal with such problems. Therefore, we also adopted the passive testing [12] in our work. The passive testing is an online test method as shown in figure 6 .

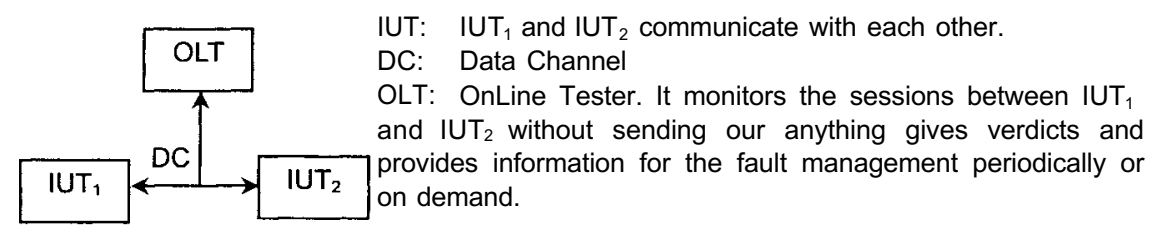

Figure 6. Model of the passive test

Compared with the active testing, the passive testing is simple in structure, while possessing more feasible and powerful abilities to perform the conformance and the interoperability tests and even analysis of the performance. Additionally, it allows more IUTs and OLTs, enabling distributed test methods. 


\subsection{Algorithms and methods for passive testing}

Algorithms to test the protocol state machine can be found in [13]. As to routing information manipulation, one generic method is topology analysis. The main idea is that correct routing information must describe the network topology correctly. We construct the topology from the routes exchanged and compare it with the actual topology. If differences are found, then there may be some errors in the routing information manipulation. Since we can get the constructed network topology from the OSPF routing information base, we mainly focused on the topology analysis for BGP-4, which can be found in [16]. Other methods like the internal process simulation are also exploited. Different modules are implemented to process the routing information collected online similarly to what the processes in the router will do. The correct scene is calculated and compared with the actual result. For BGP-4, the internal process simulation covers the generic route selection, route selection with policy constraints, and the route flap damping, etc.

\subsubsection{Testing of route selection with policy constraint}

Route selection with policy constraints is an important feature of BGP-4. These policies directly serve the relations of the Internet Service Providers (ISP) and their customers. Three basic relations are classified (clientprovider, provider-provider and customer with backup) and corresponding policies are presented. Policy conformance and violations are defined.

Definition 1. Policy conformance Conf $=<\operatorname{Conf}_{\mathrm{i}}, \mathrm{Conf}_{\mathrm{e}}>$, where $\operatorname{Conf}_{\mathrm{i}}$ is the import conformance which evaluates the process when receiving routing information. Conf $\mathrm{e}_{\mathrm{e}}$ is the export conformance which evaluates the process when advertising routing information.

Suppose $\mathrm{PS}_{\mathrm{r}}$ is the series of policies to be enforced on the received routes. $\mathrm{PS}_{\mathrm{r}}=\left[\mathrm{P}_{\mathrm{r} 1}, \mathrm{P}_{\mathrm{r} 2}, \mathrm{P}_{\mathrm{r} 3}, \ldots \ldots, \mathrm{P}_{\mathrm{m}}\right] \forall \mathrm{R}_{\mathrm{i}} \in \mathrm{R}_{-} \mathrm{RCV}, \mathrm{R}_{\mathrm{i}}^{\prime}=\mathrm{P}_{\mathrm{m}}\left(\mathrm{P}_{\mathrm{r} n-1}(\ldots \ldots\right.$ $\left.P_{r 1}\left(R_{i}\right)\right)$ ), denoted as $R_{i}^{\prime}=P_{r}\left(R_{i}\right) \cdot R_{i}^{\prime}$ is route of import conformance: $R_{i}^{\prime} \operatorname{Conf}_{I}$ $\mathrm{PS}_{\mathrm{r}}$

Suppose $\mathrm{PS}_{\mathrm{s}}$ is the series of policies to be enforced on the routes to be sent out. $\mathrm{PS}_{\mathrm{s}}=\left[\mathrm{P}_{\mathrm{s} 1}, \mathrm{P}_{\mathrm{s} 2}, \mathrm{P}_{\mathrm{s} 3}, \ldots \ldots, \mathrm{P}_{\mathrm{sm}}\right] \forall \mathrm{R}_{\mathrm{j}} \in \mathrm{R}_{-} A C P, \mathrm{R}_{\mathrm{j}}{ }^{\prime}=\mathrm{P}_{\mathrm{sm}}\left(\mathrm{P}_{\mathrm{s} m-1}(\ldots \ldots\right.$ $\left.\mathrm{P}_{\mathrm{s} 1}\left(\mathrm{~S}_{\mathrm{j}}\right)\right)$ ), denoted as $\mathrm{R}_{\mathrm{j}}{ }^{\prime}=\operatorname{PS}_{\mathrm{s}}\left(\mathrm{R}_{\mathrm{j}}\right), \mathrm{R}_{\mathrm{j}}{ }^{\prime}$ is route-of export conformance: $\mathrm{R}_{\mathrm{j}}^{\prime}$ Conf $_{\mathrm{e}} \mathrm{PS}_{\mathrm{s}}$.

Then two basic types of policy violations can be defined; other violations can be made out of these two.

Definition 2. Penetration: if $\exists R_{k} \in R \_A C P, \neg\left(\exists R_{1} \in R \_R C V, P S_{r}\left(R_{l}\right)=R_{k}\right)$, then "penetration" occurs, that is, the route is accepted after the route calculation and selection while it actually should not be.

Definition 3. Leak: if $\exists R_{k} \in R \_S N T, \neg\left(\exists R_{l} \in R_{-} A C P, P_{s}\left(R_{1}\right)=R_{k}\right)$, then "leak" occurs, that is, the route is delivered while it actually should not be. 
A separate module was implemented. With policy configurations (provided by the network operator), this module can find the basic policy violations and other subtle abnormalities.

\subsubsection{Testing of route flap damping}

Route flap is another serious problem of the Internet routing [9][17] and the "merit way" is the damping method now in use [18]. However, to our best knowledge, there is no test to evaluate the damping effect. Therefore, we worked out a simple but practical one (shown in figure 7). In this state machine, the flap of a single route, the interaction between two routes (replacement) and the condition with an alternative route are all covered. Since the route selection will finally go down to the comparison between two routes, this model is applicable for generic conditions.

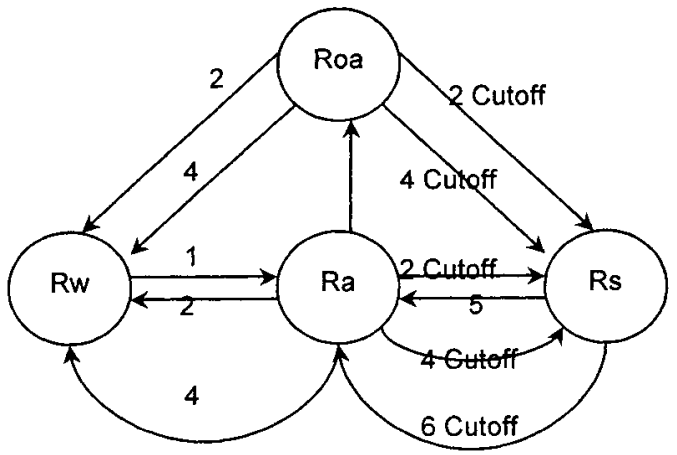

1 Recv Advertise

2 Recv Withdraw

3 Route Selected

4 Route Replaced

$5 \mathrm{TM}_{\mathrm{B}}$ Expired

6 TMc Expired

Cutoff: reached

Reuse: reached

Figure 7. State machine of route flap damping

The flap module follows this state machine and performs the merit value calculation (increase and decay). Therefore, not only can the passive testing find the existence of the route flap and its severity, it can also evaluate the effect of the route flap damping.

\section{IMPLEMENTATIONS AND TEST ACTIVITIES}

\subsection{PITS with ERI}

The basic channel can support up to 8 ports and two extended channels: the auxiliary information and the configuration channels. The auxiliary information channel is set up to access the information needed in the routing protocol test in its session with the SNMP; the configuration channel connects through telnet and configures the routing protocol and the router with corresponding commands. A test case is shown in figure 8 . 


\begin{tabular}{|c|c|c|c|c|c|}
\hline \multicolumn{6}{|c|}{ Dynamic Part } \\
\hline \multicolumn{2}{|c|}{ Test Case Name: } & \multicolumn{4}{|c|}{ UPDATE_ALL_IBGP } \\
\hline \multicolumn{2}{|c|}{ Group: } & \multicolumn{4}{|c|}{ bgp-4/IBGP/UPDATE ALL IBGP } \\
\hline \multicolumn{2}{|c|}{ Purpose: } & \multicolumn{4}{|c|}{ Test the information manipulation of IBGP } \\
\hline \multicolumn{2}{|c|}{ Default: } & \multicolumn{4}{|c|}{ NULL } \\
\hline \multicolumn{2}{|c|}{ Comments: } & \multicolumn{4}{|l|}{ NULL } \\
\hline No & \multicolumn{2}{|c|}{ BehaviorDescription } & Label & Constraints Ref & Verdict \\
\hline 1 & \multicolumn{2}{|c|}{ T! TO CONF } & & C TO CONF & \\
\hline 2 & \multicolumn{2}{|c|}{ +ESTABLISHED } & & & \\
\hline 3 & \multicolumn{2}{|c|}{ T! UPDATE01 } & & C UPDATE01 & \\
\hline 4 & T! UPDA & & & C2_UPDATE01 & \\
\hline 5 & \multicolumn{2}{|c|}{ START T_HOLD } & & & \\
\hline 6 & \multicolumn{2}{|c|}{ T? FROM_AUX } & & C FROM AUX & \\
\hline 7 & \multicolumn{2}{|c|}{ STARTT KEEP } & & & \\
\hline 8 & \multicolumn{2}{|c|}{ T?KEEP_ALIVE01 } & & C_KEEPALIVE01 & PASS \\
\hline 9 & \multicolumn{2}{|c|}{ ? TIMEOUT } & & & FAIL \\
\hline 10 & \multicolumn{2}{|c|}{ T? OTHERWISE } & & & FAIL \\
\hline 11 & \multicolumn{2}{|c|}{ ?TIMEOUT } & & & FAIL \\
\hline 12 & \multicolumn{2}{|c|}{ T? OTHERWISE } & & & FAIL \\
\hline
\end{tabular}

Figure 8 An example of a test case

In this case, the configuration channel and the auxiliary information channel are exploited. This example also demonstrates how the test activity is described integrally and uniformly.

In the acceptance sub layer, a buffer of $1600 \mathrm{X} 40$ bytes is set aside. There is no packet loss in a $10 \mathrm{M}$ or $100 \mathrm{M}$ Ethernet environment.

\subsection{Online intelligent analyzer}

The intelligent online BGP-4 Analyzer [20] is our first application of the passive testing. Compared with earlier work, this analyzer offers more efficient means for both the protocol test and the network management. It can test the state machines of BGP peers', from which some conclusions on the conformance and the interoperability can be drawn. As to the routing information manipulation, the analyzer simulates internal processes, such as the route advertisement and route withdrawal, the policy monitoring and the flap damping. The analyzer can be set to focus on some specified routing information or on certain BGP speakers' behavior. It can also collect statistics and provide basic performance parameters regarding the frequency and the amount of the data interaction. Also, the analyzer can re-display the procedure, making it easy to analyze and eliminate faults. Further, it can generate a TTCN-formatted test suite with the recorded data. Besides, it has a friendly graphical user interface (GUI) running on Linux and can attend to multiple NICs simultaneously. Data is stored in a compressed form in consideration of working over a long period time. 


\subsection{Online test system}

As a more generic system for all the routing protocols and other distributed protocols, we have implemented the prototype of an online test system [21]. The framework is shown in figure 9. There are five main components. The input component includes a protocol state machine description module, a protocol packet structure description module and a set condition module. The communication component includes a communication interface module and a condition check and filter module, which shields the difference among communication protocols of different layers. The condition check and filter module further processes the data collected and provides the test component with a uniform interface. The test component is the kernel of the OLTS, including the protocol state machine test module, the internal process simulation module and the statistics module. The cooperation component is used to organize multiple OLTS instances. The test result module, the LOG module and other modules belong to the conclusion and presentation component, which composes a test report, provides graphical presentations and generates the test suite.

For each protocol to test, a specific communication interface module is coded, descriptions of the protocol state machine and the protocol packet structure are input and the necessary internal process simulation module is applied or implemented. OLTS is implemented on a Sun Ultra 1 - Solaris and a x86-Linux separately in order to widen its application and verify its portability

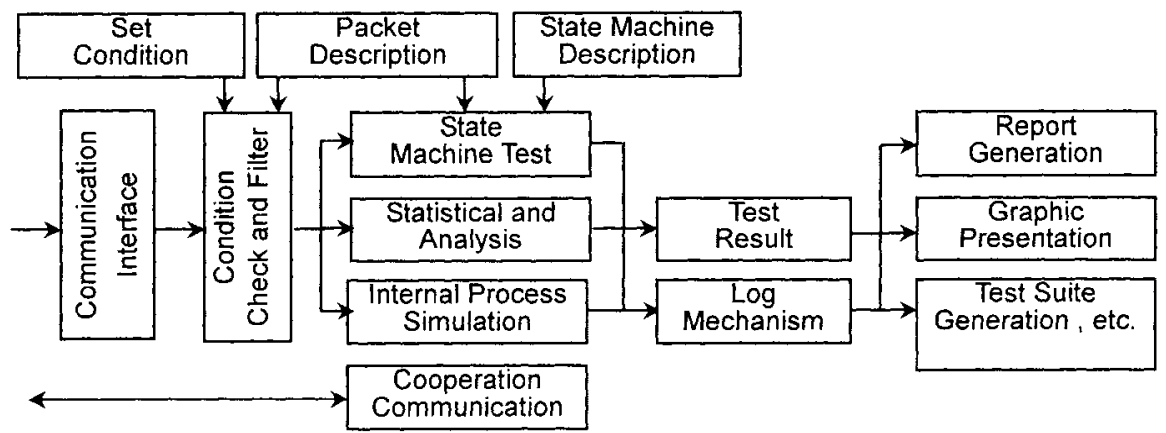

Figure 9. Framework of OLTS

Specific modules will be implemented for different communication media to shield the difference of the lower level. For example, the interface has been implemented for the most popular Ethernet, which is able to capture all the data of upper level protocols. On x86-Linux, the packet socket is exploited and DLPI (Data Link Provider Interface) [22] operating / dev/le on the Sun Ultra 1 - Solaris. For other communication media, there has been no difficulty encountered so far (even for a non-broadcast point to point link). 
Based on this, the virtual network interface (VNI) is further implemented. VNI aims to provide a uniform interface that the main process can operate. When new communication media is introduced, the only requirement is to map its interface to the uniform interface.

The internal process simulation is done via a module stack; the modules can be pushed onto the stack for different protocols and test purposes.

In consideration of testing in a distributed environment, Simple Cooperation Protocol (SCP) [23] is defined for the cooperation component, which enables multiple instances of OLTS's to work together in different places of the network. We still have a tool set, including the packet composer. It can be controlled through a control channel by other systems such as PITS.

One of the most interesting aspects is that the active testing and the passive testing are combined. The active testing and the passive testing have both advantages and limitations of their own. The active testing can send out the desired packets; the passive testing can work in production field over a long time. By combining the active testing and the passive testing, we can expect a better efficiency.

An example is the erroneous session shown in figure 10. It is first captured by the analyzer. 166.111.69.91 is one port address of the commercial router and 166.111.69.75 is that of the Router II. This session shows that due to the incorrect implementation of BGP-4 of Router II, UPDATE packets are sent out repeatedly instead of OPEN after the TCP connection is established. But the response of the commercial router is not correct either. After it responds with'a NOTIFICATION packet (line 2 in figure 10), it answers with the same UPDATE (line 5 in figure 10), which contradicts the protocol specification and leads to problems of the conformance and the interoperability along with the extra cost of the network bandwidth.

\begin{tabular}{|c|c|c|c|c|}
\hline \multicolumn{5}{|c|}{ 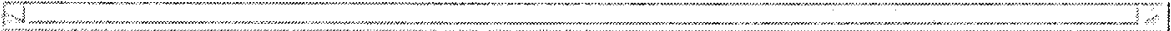 } \\
\hline 166.111 .69 .75 & 166.111 .6991 & 12/24 17:21:39 & BGP_UPDATE & 3 \\
\hline 166.111 .69 .91 & 166.111 .69 .75 & $12 / 24 \quad 17: 21: 39$ & BGP_NOTIFY & \\
\hline 166.111 .69 .75 & 166.111 .69 .91 & 12R4 17:21:49 & BGP_UPDATE & \\
\hline 166.111 .69 .91 & 166.111 .69 .75 & $12 R 21 / 221: 49$ & BGP_UPDATE & \\
\hline 166.111 .69 .75 & 166.111 .69 .91 & $12 R 4 \quad 17: 21: 54$ & BGP_UPDATE & \\
\hline 166.111 .69 .75 & 166.111 .69 .91 & $122417: 22: 9$ & BGP_UPDATE & \\
\hline
\end{tabular}

Figure 10 An erroneous session recorded

Then this fault was confirmed with the active testing and several other similar faults were uncovered with this indication. 
The test structure used in the policy-based routing test is shown in figure 11. $\mathrm{BG}_{\mathrm{t}}$ is a real router, while $\mathrm{W}_{1}$ and $\mathrm{W}_{2}$ are two online test systems. $\mathrm{N}_{1}$ and $\mathrm{N}_{2}$ are two nodes, each of which may be a real router or an active test system. $\mathrm{N}_{1}$ sets up an EBGP connection with $\mathrm{BG}_{\mathrm{t}}$ and $\mathrm{N}_{2}, \mathrm{BG}_{\mathrm{t}}$ an IBGP connection, $\mathrm{W}_{1}$ watches the session on EBGP and $\mathrm{W}_{2}$ on IBGP. With different configurations, variant tests can be performed. In a production field, $\mathrm{N}_{1}$ and $\mathrm{N}_{2}$ are all real routers, online test systems $\mathrm{W}_{1}$ and $\mathrm{W}_{2}$ monitor if there is any leak or penetration. Under the constructed test environment, $\mathrm{N}_{1}$ and $\mathrm{N}_{2}$ are test systems and they can actively test policy-based routing to detect violations.

The test structure of the route flap damping (shown in figure 12) also covers both the active testing and the passive testing. There are three types of nodes in this structure: flap source nodes, router nodes and detector nodes. The router nodes construct a network. The flap source nodes inject flap into the network at different positions and the detector collects the information about the flap damping. The test is in an active one. However, if the network is a real network, it will generate flap itself. Then the set of flap source nodes can be empty and the test switches into a passive mode.

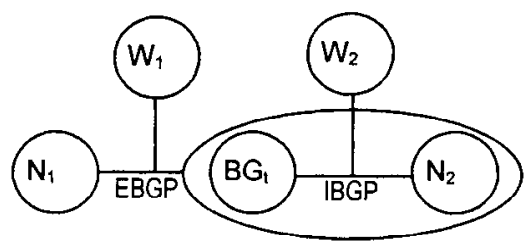

Figure 11 Test structure for policy based routing test

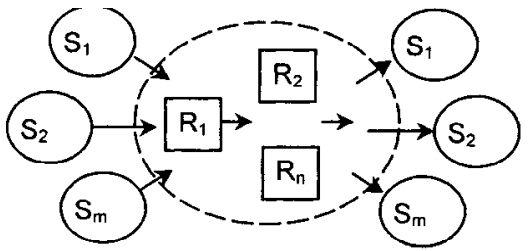

Figure 12 Test structure for route flap damping

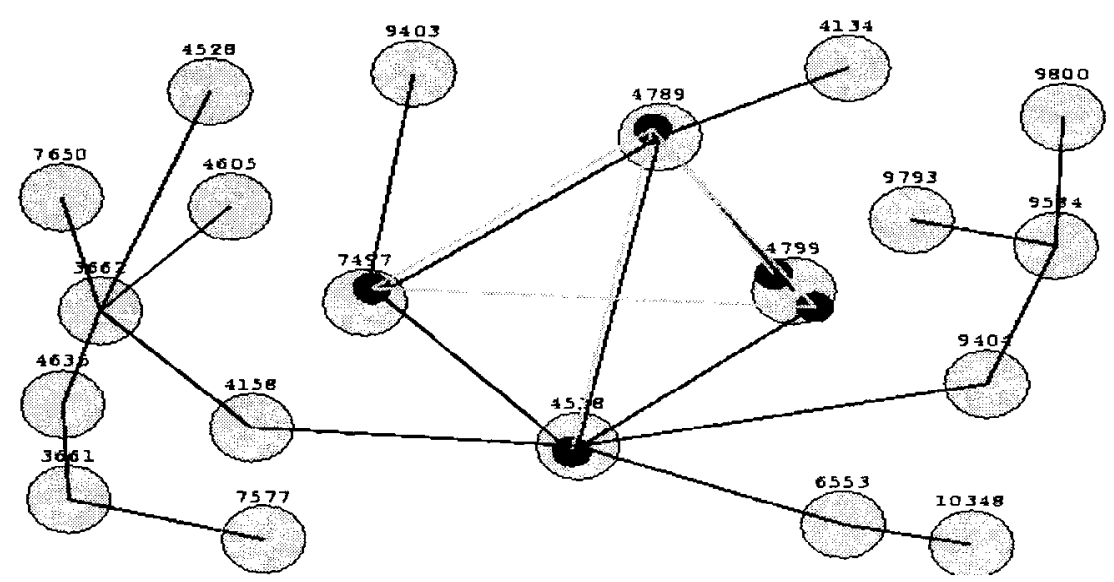

Figure 13 Topology constructed with the data collected from the backbone of CERNET 
With both the active testing and the passive testing, a large number of test activities have been performed. We have tested some commercial routers in our lab and we have performed the test on the backbone of CERNET [24]. Figure 13 is the topology constructed with the data collected on CERNET. We compare this constructed topology with the actual one to discover errors in routing information manipulation. We also offer test support to the development of routers. Their pass ratio is considerably promoted and therefore the quality is improved accordingly.

\section{CONCLUSIONS}

Despite the great importance of routing protocols, test activity is still quite limited compared with the testing of communication protocols. In addition to our previous work, reference [11] discusses the testing of IP routing protocols in the environment generated by software tools. Craig Labovitz has collected the routing data for two years and published several papers [17]. Currently there are some commercial test systems, such as HP Router Tester [25] and NetCom Systems Smartbits [26], etc. They mainly focus on the performance of the system. They are all active testers and possess a high degree of industrialization. The price is rather expensive and they are always difficult to extend. For example, users can't develop test suites for new protocols.

This paper presents our efforts to cover important features of Internet routing protocols. However, there is still quite a lot to do. Routing protocols are still progressing, while models, methods and other techniques still need further refinement. At the same time, more efforts must be expended within the field of the protocol test, through which the validity of new methods can be proved and new problems can be discovered.

\section{REFERENCES}

[1] C. Hedrick. Routing Information Protocol. RFC 1058. 1988

[2] J. Moy. OSPF Version 2. RFC 2178. 1998

[3] Y. Rekhter, T. Li. A Border Gateway Protocol 4 (BGP-4). RFC 1771, 1995

[4] ISO,Open Systems Interconnection (OSI) Routing Protocol, 1985

[5] Cisco Systems, Inc. Interior Gateway Routing Protocol, 1987

[6] Cisco Systems, Inc., Enhanced Interior Gateway Routing Protocol, 1989

[7] Jianping $\mathrm{Wu}$, Xiuhuan Chen, Ruibing Hao et al. PITS-The protocol integrated test system based on formal technology, Journal of Tsinghua Univ. 1998: 38(S1):26 29. 
[8] T. G. Griffin, G. Wilfong, An Analysis of BGP Convergence Properties, Proc of Sigcomm 99, 1999

[9] C. Labovitz, G.R. Malan, F. Jahanian. Internet routing instability. IEEE/ACM Transactions on Networking Oct 1998 IEEE Piscataway NJ USA 1063-6692 IEANEP, 1998; 6: 515-527

[10] Yixin Zhao, Jianping Wu, Xia Yin and Bo Han. Test of BGP-4 based on the Protocol Integrated Test System. Proc of APCC2000. Korea. 2000

[11] Ruibing Hao, D. Lee, K. Rakesh. et al. Testing IP Routing Protocols - From Probabilistic Algorithms to a Software Tool. KLUWER ACADEMIC PUBLISHERS. Proc of FORTE/PSTV2000. Pisa, Italy. 2000.

[12] D. Lee, A.N. Netravali, K.K. Sabnani et al. Passive Testing and Applications to Network Management, Proc of ICNP 97,1997

[13] Yinin Zhao, Jianping Wu. Algorithm to determine states applied in online test. Chinese Journal of Electronics. 2000; 11: 33-40

[14] Yixin Zhao, Bo Han, Xia Yin and Jianping Wu. Study of Routing Protocols Test. Computer Engineering.2000; 26:769-774

[15] ISO. Information Technology, Open System Interconnection, Conformance Test Methodology and Framework. IS-9646, ISO, 1991

[16] Yixin Zhao, Xia Yin, Jianping Wu et al. Fault Management of Inter-domain Routing with Passive Test. Proc of ICT2001. Romania, Bucharest, 2001

[17] C. Labovitz, R. Wattenhofer, S. Venkatachary. The Impact of Internet Policy and Topology on Delayed Routing Convergence. Tech. Report. 2000

[18] C. Villamizar, R. Chandra, R. Govindan. BGP Route Flap Damping. RFC 24391998

[19] Yixin Zhao, Xia Yin, Bo Han and Jianping Wu. Enhanced Reference Implementation Applied in Routing Protocol Test. Proc of ICACT 2001, Korea, 2001

[20] Yixin Zhao, Jianping $\mathrm{Wu}$ and Bin Yu. Intelligent Online BGP-4 Analyzer. IEEE Computer Society. Proc of MASCOTS 2000. Los Vaqueros Circle: IEEE Computer Society, 2000

[21] Yixin Zhao, Xia Yin, Bo Han and Jianping Wu. The Online Test System Applied in the Test of Routing Protocols. High Technology Letters (In press).2000

[22] OSI. Data Link Provider Interface Specification, UNIX International OSI Work Group, revision 2.0.0. 1991

[23] Yixin Zhao. Study of BGP-4 conformance test. Master thesis. Tsinghua Univ. 2000

[24] China Education and Research Network CERNET. http://www.cernet.edu.cn

[25] Agilent Technologies. http://www.tm.agilent.com/. 1999

[26] Spirent Communications, Inc. http://www.netcomsystems.com. 2000 